\title{
Suicidality and Psychological Adverse Events in Patients Treated with Finasteride
}

\author{
Ralph M. Trüeb ${ }^{a}$ Maria Fernanda Reis Gavazzoni Dias ${ }^{b} \quad$ Hudson Dutra Rezende ${ }^{c}$ \\ ${ }^{a}$ Center for Dermatology and Hair Diseases Professor Trüeb, Wallisellen, Switzerland; ${ }^{b}$ Department of Dermatology, \\ Universidade Federal Fluminense, Centro de Ciências Médicas, Hospital Universitário Antonia Pedro, Niteró, Rio de Janeiro, \\ Brazil; ' Department of Dermatology, Alvaro Alvim School Hospital, Campos dos Goytacazes, Rio de Janeiro, Brazil
}

\section{Keywords \\ Male androgenetic alopecia · Oral finasteride . \\ Psychological adverse events · Suicidality · Post-finasteride syndrome}

Hysteria Is Only Possible with an Audience Chuck Palahniuk

Nguyen et al. [1] recently published their investigation into suicidality and psychological adverse events in male patients treated with oral finasteride. They provide the results of a pharmacovigilance case-noncase study based on a disproportionality analysis to detect signals of adverse reaction of interest reported with finasteride in $\mathrm{Vi}$ giBase, the World Health Organization's global database of individual case safety reports. A significant disproportionality signal for suicidality and psychological adverse events in finasteride was identified. In sensitivity analyses, younger patients with androgenetic alopecia (AGA) had significant disproportionality signals for increased suicidality, while such signals were not detected in older patients with benign prostatic hyperplasia. Sensitivity analyses also showed that the reports of these adverse events significantly increased after 2012, when reports

karger@karger.com

(c) 2021 S. Karger AG, Basel

www.karger.com/sad

Karger! started emerging on men who had used finasteride and either attempted or completed suicide.

In fact, Altomare and Capella [2] were among the first to report depression circumstantially related to the administration of finasteride for treatment of AGA in the dermatologic literature as early as 2002 and to urge further studies on their observation. Only with the emergence of the post-finasteride syndrome (PFS) in 2012 [3] has finasteride-induced depression found the due attention of the medical community, with the respective reverberation on social media.

In Skin Appendage Disorders, Fertig et al. [4] and Trüeb et al. [5] published on the matter from the perspective of dermatologists specialized in treating hair loss, who have the most clinical experience with oral finasteride for treatment of male AGA. Although individuals suffering from PFS present with homogenous symptoms of persistent sexual, mental, and physical side effects despite stopping finasteride, the condition is as yet not recognized by the scientific community as a valid nosologic entity of somatic or endocrine origin. Specifically, Fertig et al. [4] scrutinized the available literature on PFS, and did not find the concept of PFS to be plausible, on the grounds that it emerged from reports of non-dermatolo- 
gists and neuroendocrinological research, and so far it has only been documented in low-quality studies with a strong bias selection and a significant nocebo effect [6].

In our long-standing dermatological practice with frequent finasteride prescriptions, we have rarely observed depression or suicidality and only a single case of PFS. In our experience, sexual side effects, such as decreased libido and erectile dysfunction, as well as testicular tenderness and (rarely) gynecomastia have been reversible upon cessation of oral finasteride treatment, and without any sequelae. This is consistent with the respective data on safety and efficacy of $1 \mathrm{mg}$ oral finasteride in the so far largest study of 3,117 Japanese men treated for AGA published in 2012 [7].

Eventually, after 20 years of uneventful practice in prescribing oral finasteride for male AGA, we published our first case observation of PFS, which we interpreted as an induced delusional disorder on a background of a histrionic personality disorder with the potential for mass psychogenic illness (hysteria) [5]. With this, we acknowledge the existence of the condition, albeit on alternative leads from the current understanding. Ultimately, patients with PFS are unlikely to benefit from hormonal treatments, since no evidence of androgen deficiency, persistent steroid 5-alpha-reductase inhibition, or androgen insensitivity has so far consistently been identified. Attention must be focused rather on the associated psychopathological disorder with appropriate psychotherapy and specific psychotropic agents depending on the underlying mental disorder (depressive, delusional, or somatoform disorder). So far, there are no predictive factors for the psychological adverse effects of finasteride and the PFS; nevertheless, we suggested that a preexisting mental health disorder may put patients at an increased risk [5].

The psychological effects of alopecia may be hard to differentiate from preexisting psychopathology. However, patients with personality disorders tend to experience more distress from alopecia than non-disordered patients, since these individuals lack a secure sense of self and effective coping skills, and therefore may be especially vulnerable to the adverse effects of both alopecia and the media coverage on finasteride and adverse effects. Indeed, Maffei et al. [8] found the prevalence of personality disorders in subjects with AGA to be significantly higher than in the general population.

Personality disorders are characterized by deeply ingrained maladaptive patterns of behavior and personality style that are sufficiently rigid and deep-seated to bring a person into repeated conflicts with the social environ- ment. Personality disorders have their onset in adolescence or early adulthood and may cause significant emotional suffering by virtue of difficulties in social relationships and performance, that is, Maffei et al. [8] identified 3 distinct personality profiles in males presenting to the physician with the complaint of hair loss from AGA: (1) suspicious, with grandiose sense of self-importance, obsessive, and socially withdrawn; (2) impulsive, identity disordered, and socially maladjusted; and (3) dramatic, emotional, and dependent [8]. It would therefore seem appropriate to ascertain a personal history or screen for preexisting mental health issues, specifically depressive disorder or histrionic personality disorder, for a stringent selection of patients before starting them on finasteride, since these may put patients at an increased risk of developing emotional disorders, such as depression, and somatization, such as the PFS.

Ultimately, the doctor's choice has to be consistent with the patient's philosophy of living, particularly when prescribing a drug for treatment of a cosmetic condition, where a choice must be made for long-term systemic medication with the risks of sexual side effects, depression, and PFS. Under these circumstances, in the setting of a dermatologic practice, we have fared well in successfully treating young males with AGA with oral finasteride with a low frequency of mood disorders and sequelae for the last 20 years.

\section{Conflict of Interest Statement}

The authors have no conflicts of interest to disclose.

\section{Funding Sources}

The authors did not receive any funding.

\section{Author Contributions}

Professor Trüeb takes responsibility for the integrity of the data and the accuracy of the data analysis. Study concept and design: not applicable. Acquisition, analysis, and interpretation of data: not applicable. Drafting of the manuscript: Trüeb, Gavazzoni, and Dutra. Critical revision of the manuscript for important intellectual content: Trüeb, Gavazzoni, and Dutra. Statistical analysis: not applicable. Obtained funding: not applicable. Administrative, technical, or material support: not applicable. Study supervision: not applicable. 


\section{References}

1 Nguyen DD, Marchese M, Cone EB, Paciotti $\mathrm{M}$, Basaria S, Bhojani N, et al. Investigation of suicidality and psychological adverse events in patients treated with finasteride. JAMA Dermatol. 2020 Nov 11:e203385.

2 Altomare G, Capella GL. Depression circumstantially related to the administration of finasteride for androgenetic alopecia. J Dermatol. 2002;29(10):665-9.

3 Irwig MS. Depressive symptoms and suicidal thoughts among former users of finasteride with persistent sexual side effects. J Clin Psychiatry. 2012;73(9):1220-3.
4 Fertig R, Shapiro J, Bergfeld W, Tosti A. Investigation of the plausibility of 5-alpha-reductase inhibitor syndrome. Skin Appendage Disord. 2017;2(3-4):120-9.

5 Trüeb RM, Régnier A, Dutra Rezende H, Gavazzoni Dias MFR. Post-finasteride syndrome: an induced delusional disorder with the potential of a mass psychogenic illness? Skin Appendage Disord. 2019;5(5):320-6.
6 Mondaini N, Gontero P, Giubilei G, Lombardi G, Cai T, Gavazzi A, et al. Finasteride $5 \mathrm{mg}$ and sexual side effects: how many of these are related to a nocebo phenomenon? J Sex Med. 2007;4(6):1708-12.

7 Sato A, Takeda A. Evaluation of efficacy and safety of finasteride $1 \mathrm{mg}$ in 3177 Japanese men with androgenetic alopecia. J Dermatol. 2012;39(1):27-32.

8 Maffei C, Fossati A, Rinaldi F, Riva E. Personality disorders and psychopathologic symptoms in patients with androgenetic alopecia. Arch Dermatol. 1994;130(7):868-72. 